\title{
A Comparative Study of Dislocations in HVPE GaN Layers by High-Resolution X-Ray Diffraction and Selective Wet Etching
}

\author{
Vladimir Ivantsov and Anna Volkova \\ GaN Lab, Ostendo Technologies, Inc., 12214 Plum Orchard Drive, Silver Spring, MD 20783, USA \\ Correspondence should be addressed to Vladimir Ivantsov, vladimir.ivantsov@ostendo.com \\ Received 18 June 2012; Accepted 31 July 2012 \\ Academic Editors: A. Oyamada, M.-H. Phan, and H.-D. Yang
}

Copyright ( 2012 V. Ivantsov and A. Volkova. This is an open access article distributed under the Creative Commons Attribution License, which permits unrestricted use, distribution, and reproduction in any medium, provided the original work is properly cited.

It has been shown during the present study that the E-etching at elevated temperatures can be adopted for the dislocation etching in hydride vapor-phase epitaxy (HVPE) GaN layers. It has been found that the X-ray diffraction (XRD) evaluation of the dislocation density in the thicker than $6 \mu \mathrm{m}$ epilayers using conventional Williamson-Hall plots and Dunn-Koch equation is in an excellent agreement with the results of the elevated-temperature E-etching. The dislocation distribution measured for 2-inch GaN-onsapphire substrate suggests strongly the influence of the inelastic thermal stresses on the formation of the final dislocation pattern in the epilayer.

\section{Introduction}

It is the optoelectronic properties that make GaN extremely attractive for a wide range of applications. It is needless to say that such defects as dislocations can adversely influence them. The high-resolution XRD is able to provide measurements of the dislocation densities in a nondestructive way. Therefore the XRD can be considered as the preferred metrology technique for monitoring of product substrates. Despite a number of the XRD methods developed for evaluation of the dislocation density [1], they are far from having reached conclusive results from a metrological point of view. Recently developed selective wet etching of the dislocations in bulk GaN has been confirmed to show the strict correspondence between the etch pits density (EPD) and the dislocation density measured by transmission electron microscopy (TEM) [2].

The purpose of this study is to examine the relationship between the etch pit density after selective wet etching and the dislocations density measured by the XRD in mosaic crystal approximation for the HVPE GaN epilayers grown on sapphire substrates.

\section{Selective Wet Etching Experiments}

Among various etching techniques aiming to be used for dislocation counting in $\mathrm{GaN}$, the selective wet etching with a molten eutectic flux of $\mathrm{KOH}$ and $\mathrm{NaOH}$ (dubbed E-etchant) has the most prominent foundation to be considered as the most appropriate. This technique has been adopted for the etching part of the present study. Both intentional introduction of dislocations by indentation and TEM measurements confirmed direct correspondence of the etch pits to dislocations in bulk GaN $[3,4]$. Application of the Eetching to the epitaxial $\mathrm{GaN}$ has been found to show that the edge dislocations could be missed on etching pattern [3]. It was suggested in [5] that the major factor influencing etching result could be the etching temperature and this temperature has required careful consideration and research.

Whole 2-inch diameter GaN-on-sapphire substrates as well as their parts have been used as samples for the study. The thicknesses of the HVPE epilayers ranged from 5 to $21 \mu \mathrm{m}$. Both undoped and Mg- or Si-doped samples have been tested. The samples were wiped clean with distilled water and acetone and placed face-up in a platinum wire 


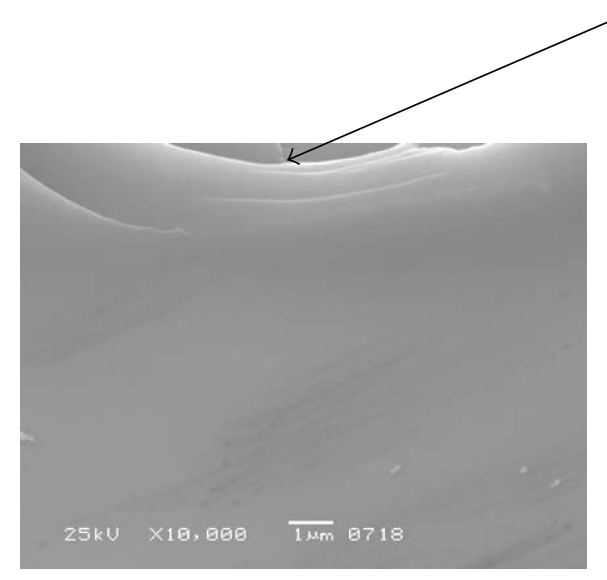

(a)

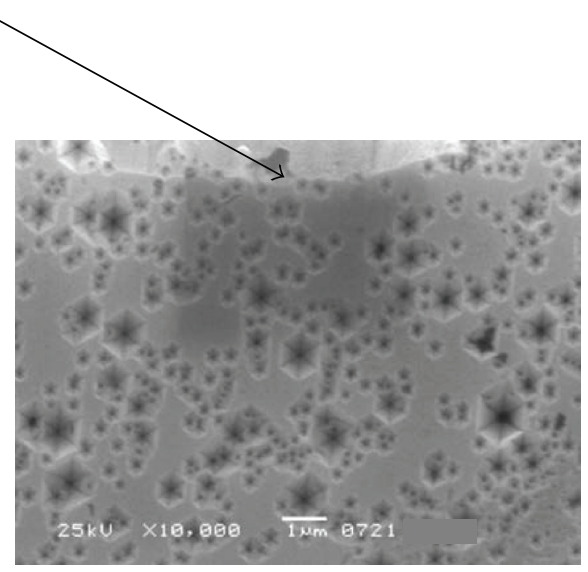

(b)

Figure 1: As-grown surface of $\mathrm{GaN}$ epilayer before (a) and after (b) 3-min etching in $\mathrm{KOH}+\mathrm{NaOH}$ eutectic flux (E-etchant) at $540^{\circ} \mathrm{C}$. Only hexagonal-shaped etch pits are observed. The EPD in (b) is $3.4 \times 10^{8} \mathrm{~cm}^{-2}$. The arrows denote the reference dent at the edge of the substrate. The darkish area in (b) is an artifact of the SEM exposition.

tray. To verify the influence of $\mathrm{Cl}^{-}$ions on the etch pattern, $\mathrm{NaCl}$ has been added from time to time to the E-flux at no more than 5 mole\% level (EI-etchant). The etchant has been heated, dried, and eventually melted in thermally shielded nickel crucible mounted on the top of a hot plate. Since temperature plays a critical role in the etching kinetics, special attention has been paid to the accuracy of temperature measurement. The temperature has been measured with a type-K thermocouple connected to a Cen Tech P37772 digital multimeter. The accuracy of the measurements was determined to be $\pm 0.5^{\circ} \mathrm{C}$ over the $100-600^{\circ} \mathrm{C}$ range. After the etching, samples were immediately washed in running distilled water and rinsed out with acetone. The etching patterns have been studied in scanning electron microscope (SEM) JEOL JSM-5600 at $12-25 \mathrm{kV}$ accelerating voltage and low probe current.

\section{Results of the Selective Wet Etching Experiments}

In Figure 1, the representative images of the as-grown GaN epilayer surface before and after E-etching are presented. As it was noted in $[1,2]$ for the bulk GaN samples, the shape of the pits depends on the polarity of the etched surface. The pits are hexagonal on Ga-polar and circular on N-polar basal surface. The E-etching of HVPE layers has exposed the hexagonally shaped pits only. Therefore, it has been concluded that HVPE GaN layers on sapphire substrates grow with Ga face-up, which is consistent with the result of the HVPE GaN epitaxy on GaAs substrates [6], where, as a probable cause for that peculiar feature, faster growth rate of Ga-face compared to $\mathrm{N}$-face was suggested. Growth always results in Ga-face polarity irrespective of the pretreatment of the substrate or nucleation layer used. It was shown experimentally [7] that the relation of the growth rate and $\mathrm{GaN}$ polarity is determined by the decomposition rate. At higher than $900^{\circ} \mathrm{C}$ temperatures the growth rate of the

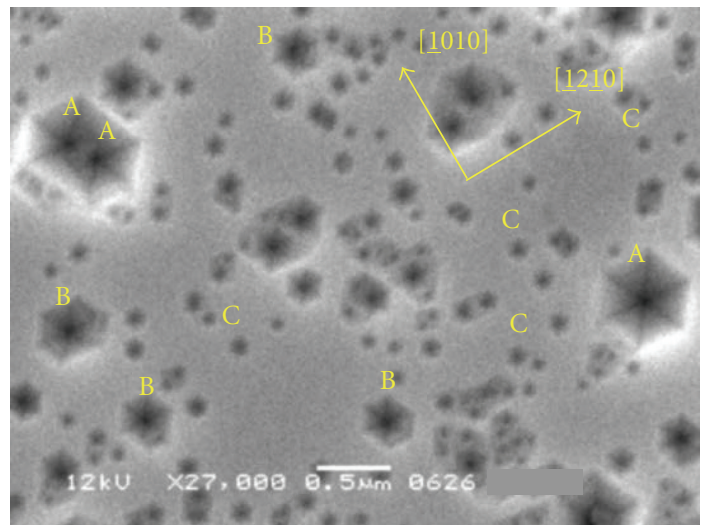

Figure 2: The etch pattern of HVPE GaN epilayer after 2-min Eetching at $540^{\circ} \mathrm{C}$. The A pits are ascribed to the screw dislocations (the $\mathrm{EPD}_{\mathrm{A}}$ is $2.8 \times 10^{7} \mathrm{~cm}^{-2}$ ), the middle-size $\mathrm{B}$ pits-to the mixed $\left(1.2 \times 10^{8} \mathrm{~cm}^{-2}\right)$, and the smallest $\mathrm{C}$ pits-to the edge dislocations $\left(8.7 \times 10^{8} \mathrm{~cm}^{-2}\right)$.

Ga-face indeed prevails that of the N-face due to the higher decomposition rate of the later one.

For the Ga-face of bulk GaN crystals the etching temperature from $350-450^{\circ} \mathrm{C}$ range was settled to be preferable [4]. Etched at these temperatures $\mathrm{GaN}$ epilayers acquired faint etching pattern with smeared shallow pits, whose density was about mid-end $10^{7} \mathrm{~cm}^{-2}$. Since there was no clear tendency of the pit size to saturate with an increase in etching time, the etching temperature has been increased significantly to the $500-600^{\circ} \mathrm{C}$ range. It was speculated afterwards that this increase has to be anticipated for the epilayers due to their compressive state in contrast to a relaxed state of bulk material. The etching at elevated temperatures resulted in an appearance of a steady pattern of the hexagonal pits of four distinctly different sizes (Figures 1, 2, and 3). The prolongation of etching time led to an enlargement of the pits but did not have a marked influence on their density. 
TABLE 1: Results of the XRD measurements and etching experiments.

\begin{tabular}{lccccccc}
\hline Sample number & $\begin{array}{c}\text { Layer thickness } \\
\mu \mathrm{m}\end{array}$ & $\begin{array}{c}\rho_{\text {XRDscrew }} \\
10^{8} \mathrm{~cm}^{-2}\end{array}$ & $\begin{array}{c}\rho_{\text {XRDedge }} \\
10^{8} \mathrm{~cm}^{-2}\end{array}$ & $\begin{array}{c}\rho_{\text {XRDtotal }} \\
(\text { XRD }) \\
10^{8} \mathrm{~cm}^{-2}\end{array}$ & $\begin{array}{c}\rho_{\text {EPDtotal }} \\
(\text { Etch) } \\
10^{8} \mathrm{~cm}^{-2}\end{array}$ & $\begin{array}{c}\rho_{\text {EPDtotal }} / \rho_{\text {XRDtotal }} \\
(\text { Etch } / \text { XRD) }\end{array}$ & $\begin{array}{c}\rho_{\text {XRDtotal }} / \rho_{\text {XRDscrew }} \\
(\text { total } / \text { screw) }\end{array}$ \\
\hline 1 (undoped) & 5.0 & 4 & 6.4 & 10.4 & 12.0 & 1.15 & 2.6 \\
2 (Mg-doped) & 6.2 & 3.3 & 6.2 & 9.8 & 10.1 & 1.03 & 3.0 \\
3 (undoped) & 16.3 & 2.0 & 5.1 & 7.1 & 7.1 & 1.00 & 3.6 \\
4 (undoped) & 21.0 & 1.1 & 3.7 & 4.8 & 5.0 & 1.04 & 4.4 \\
\hline
\end{tabular}

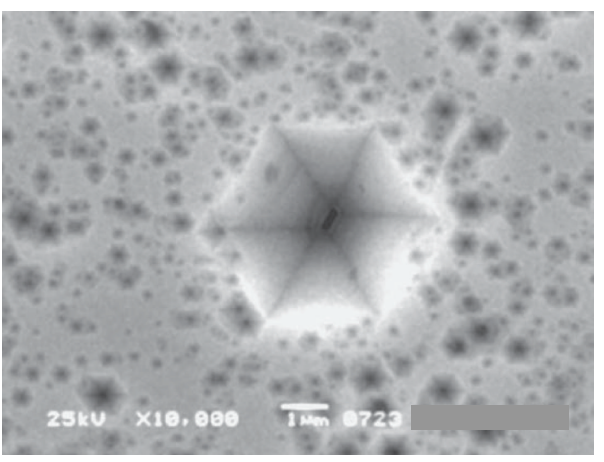

Figure 3: Nanopipe in HVPE GaN epilayer after 7 min etching at $580^{\circ} \mathrm{C}$ in EI-flux. The submicron-scale opening is visible at the bottom of the pit.

Three different sizes etch pits were observed in [8] after etching $\mathrm{GaN}$ epilayers in molten $\mathrm{KOH}$. According to the plan-view TEM observations in [3] made after E-etching, the biggest pits correspond to the hollow-core screw dislocations (dubbed nanopipes) while the mid-size ones relate to the mixed dislocations. Follow these observations and liable correlation between the size of the pit and the elastic energy of the defect; the smallest pits are associated with the perfect $1 / 3\langle 1120\rangle$ edge dislocations whose elastic energy is smaller than that of screw $\langle 0001\rangle$ and mixed $1 / 3\langle 1123\rangle$ dislocations. In Figure 2, these smallest pits (C) overwhelmingly dominate the etch pattern of the HVPE GaN epilayer.

Since the mixed dislocations are inclined in respect to the epilayer surface [9], corresponding etch pits are shaped as the medium-size slightly asymmetric hexagons (Figure 2(B)). The screw dislocations were etched at a higher rate and formed larger pits in the etch pattern (Figure 2(A)). The biggest etch pits comprise the fourth type, which is related to the nanopipes that not always, but from time to time, spoil GaN epilayers (Figure 3). The etched orifice at the bottom of nanopipe-related pit exposes the hollow core of the dislocation.

Developed for dislocation etching at elevated temperatures procedure has been used for the counting of dislocations in the HVPE GaN epilayers grown on sapphire substrates. The total density of all kinds of pits, $\rho_{\text {EPDtotal }}$, is presented in Table 1 for epilayers having different thicknesses and doping to be compared with the corresponding results of XRD measurements, $\rho$ XRDtotal .

Crystallographic orientation of the etched hexagons can be easily determined with respect to the position to the flat on (0001) substrate. The sides of the hexagons lie along $\langle 2110\rangle \mathrm{GaN}$ directions (Figure 3), the clue that could be conveniently used for quick orientation of the stripes in different type epitaxial overgrowth. The inner correspondence between crystal etching and crystal growth makes it suitable to consider etch pit like a negative crystal and ascribe to direction of the fastest growth the direction of the slowest etching. The optimal stripe orientation is better to be made normal to the direction of the fastest growth that corresponds to $\langle\underline{1010}\rangle$ direction for $c$-zone of GaN.

\section{X-Ray Diffraction Experiments}

A broadening of the XRD peak can be caused for both instrumental and sample-related reasons. Application of high-resolution X-ray diffractometry makes the instrumental broadening negligible, but the deconvolution of the sample-related components still is not defined explicitly [10]. Even in simplified methods the assumptions on the nature of the broadening are required. The attempt to apply XRD peak profile analysis to calculation of the dislocation density in HVPE GaN [11] first required modification of the existing method; second, introduced new parameters to be fitted without any physical meaning attached. Diffuse Xray scattering from epitaxial layers was used for calculation of just the screw component of the dislocation density in [12]. Developed in [13] method for peak fitting after determination of the ratio of Lorentzian and Gaussian constituents instead of full profile refinement gives for symmetric peaks a classic Williamson-Hall type analysis if containing correlation length term can be neglected (that is appropriate for the thick HVPE epilayers).

The Williamson-Hall (WH) approach [14] suggests the dominant role of size and strain-related components in the peak broadening and employs the independence of the size broadening on the Bragg angle for their separation. Due to the simplicity of the WH plot, it can always be done first to evaluate the main possible cause of broadening [15]. The validity of the assumption that the strain-related broadening is mainly caused by dislocations in HVPE GaN has been evaluated in this part of the study.

The dislocation density in HVPE GaN epilayers has been calculated in the frame of the mosaic crystal approximation. Then, screw component of the dislocation density, $\rho_{\text {screw }}$, accommodates the tilt distortion in the epilayer, $\alpha$, and, according to [16],

$$
\rho_{\text {screw }}=\frac{\alpha^{2}}{2 \pi b_{\text {screw }}^{2} \cdot \ln (2)},
$$


where $b_{\text {screw }}$ is the Burgers vector of the screw dislocation $(5.185 \AA$ in $\mathrm{GaN})$, and $2 \pi \cdot \ln (2) \approx 4.35$. The equation is assumed to be valid for random spatial distribution of the dislocations. The randomness in the distribution of the etch pits has been called to corroborate this assumption.

Similar equation has been applied for the calculation of the edge component of the dislocation density, $\rho_{\text {edge. If, again, }}$ it is assumed that the twist distortion angle, $\beta$, is exactly defined by the edge component of the dislocation density $\left(b_{\text {edge }}=3.189 \AA\right.$ in $\left.\mathrm{GaN}\right)$, then

$$
\rho_{\text {edge }}=\frac{\beta^{2}}{2 \pi b_{\text {edge }}^{2} \cdot \ln (2)} .
$$

The tilt and twist distortions have been determined using the WH plots for symmetric (000l) and skew-symmetric (101l) family reflections. The assumption of Lorentzian peak profile presumes linear summation of the broadenings driven by the dislocations and finite crystallite size. Fitting the dependence of $\mathrm{FWHM}_{\mathrm{symm}} \sin \theta / \lambda$ on $\sin \theta / \lambda$ for the family of symmetric (0002), (0004), and (0006) reflections with a linear function provides the tilt angle, $\alpha$, that is equal to $\alpha=\tan ^{-1}$ (slope).

The analogous extrapolation procedure has been used for assessment of the twist angle, $\beta$, caused by the edge component of the dislocation density. The procedure has included $\omega$-scans of the out-of-plane skew-symmetric (1012), (10113), (1014), and (1015) reflexes, and deriving the slope of the $\mathrm{WH}$ plots in $\mathrm{FWHM}_{\text {asymm }} \sin \theta / \lambda-(\cos \chi \cdot \sin \theta) / \lambda$ coordinates. It is worth noting that, being completely accurate, the use of the FWHMs instead of integral breadths is more appropriate for Gaussian distribution. Nevertheless, from the practice, if only the Lorentzian distribution is accounted for the sizebroadening, the Gaussian broadening can be represented as strain broadening, the fact that is related to the real peak profile, which is neither Lorentzian nor Gaussian but is a convolution of the two.

The Rigaku ATX-E diffractometer with double-crystal Ge (220) channel-cut monochromator and $0.05 \times 10 \mathrm{~mm}^{2}$ divergence slits has been employed for XRD measurements. The X-ray tube, supplied with $50 \mathrm{kV}$ voltage and $30 \mathrm{~mA}$ current, in combination with a graded multilayer mirror has provided a beam with $0.1 \mathrm{mrad}$ divergence at $\mathrm{CuK}_{\alpha 1}$ $(1.5406 \AA)$ radiation. The accuracy of the XRD measurements was evaluated from the multiple measurements at the same sample spot. For the symmetric reflections it was determined to be within $5 \%$, while for the skew-symmetric reflections it was $7 \%$.

\section{Results of the XRD Measurements}

In Figures 4 and 5 the illustrative results of the WH plot drawings for symmetric and skew-symmetric reflections measured on HVPE Si-doped GaN epilayer are presented. Thickness of the epilayer and donor concentration in it (according to capacitance-voltage examination) have been measured to be $5.2 \mu \mathrm{m}$ and $1.7 \times 10^{17} \mathrm{~cm}^{-3}$, correspondingly. Fitting the data and refining the parameters of the plots were provided with the assistance of a simple MathCAD program.

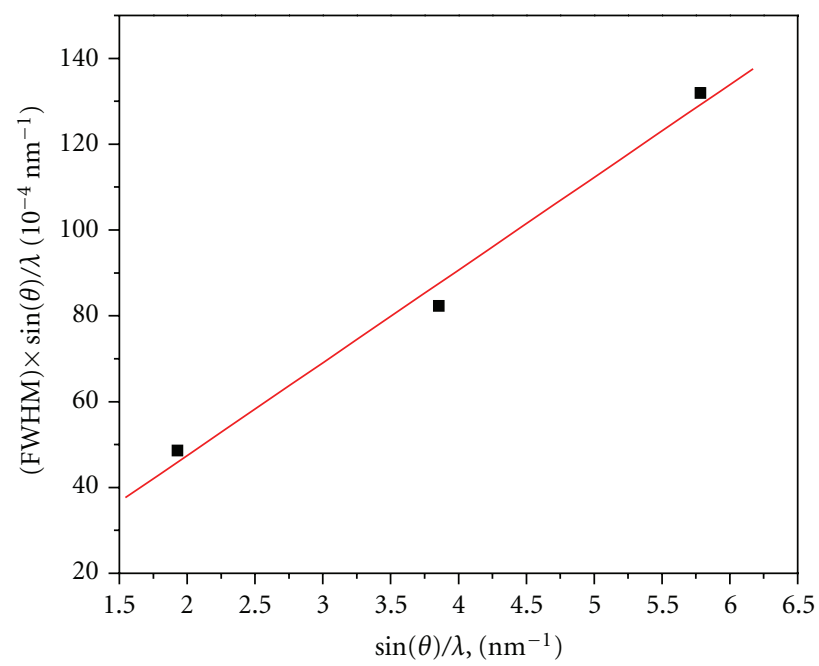

FIGURE 4: Williamson-Hall plot for symmetric (00.2), (00.4), and (00.6) reflections of HVPE GaN on sapphire. Thickness of the epilayer is $5.2 \mu \mathrm{m}$. The tilt angle is $2.16 \mathrm{mrad}$. The volume-weighted correlation length is $\sim 1 \mu \mathrm{m}$.

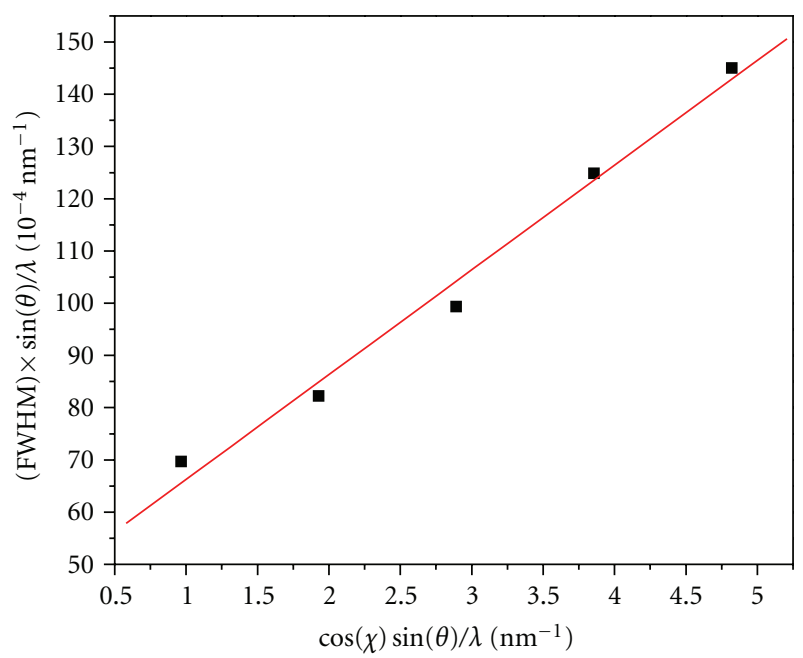

FIgURE 5: Williamson-Hall plot for the skew-symmetric (10.1), (10.2), (10.3), (10.4), and (10.5) reflections of HVPE GaN on sapphire (the same sample and spot as in Figure 4). The twist angle is determined to be $2.03 \mathrm{mrad}$.

The twist and tilt angles derived from the plots have been substituted into (1) and (2) to calculate screw and edge components of the dislocation density. The volume-weighted correlation length has been determined along with the angles from the extrapolated plot intercept with the ordinate axis. It is shown in figure caption. The above procedure has been applied to samples with different GaN thicknesses and dopings in order to be used for the comparative analysis of the XRD and etching results. E-etching of the samples and etch pits counting have been carried out after the XRD measurements at the locations where the measurements were done. The results of the XRD measurements and etching experiments are presented in Table 1. 


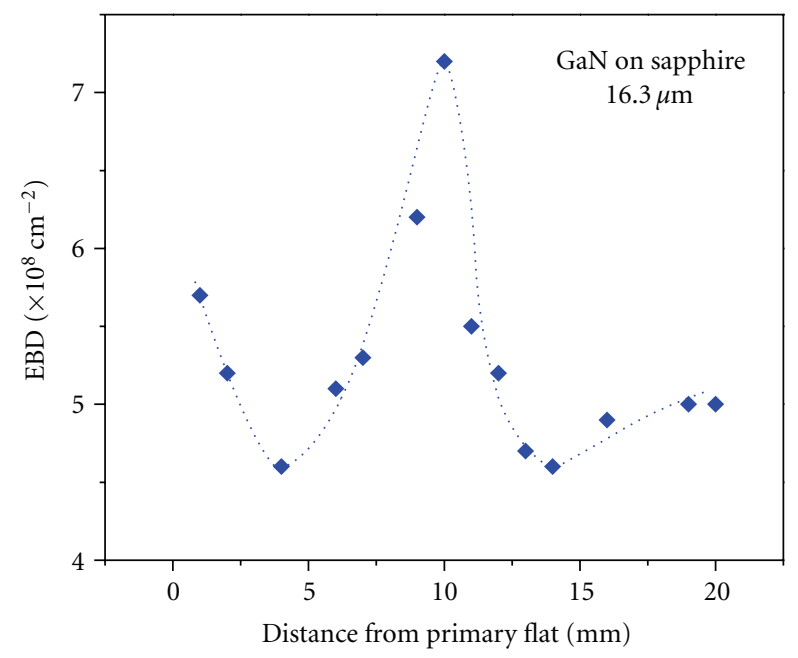

Figure 6: Dependence of the dislocation density on the distance from the flat along the radius of GaN-on-sapphire substrate.

For all studied HVPE GaN epilayers with different thicknesses from $5 \mu \mathrm{m}$ to $21 \mu \mathrm{m}$ data analysis have shown strong correspondence between the EPDs after E-etching and dislocation densities determined from the XRD WH measurements. In fact the difference between the both does not exceed $15 \%$ and becomes much smaller $(<5 \%)$ for the thicker epilayers. Both EPD and XRD values have tendency to decrease with the epilayer thickness slowly so that even for $20 \mu \mathrm{m}$ thick epilayer, threading dislocations still present in it at around $10^{8} \mathrm{~cm}^{-2}$ density. Obviously, grown on sapphire low-dislocation density moderately thick HVPE GaN requires additional dislocation-stopping methods in order to be accepted as a laser material. (As it has been recently found by the authors of the present paper, it is quite feasible even for $\sim 10 \mu \mathrm{m}$ thick epilayers.) The results of the study demonstrate that the decrease in dislocation density with epilayer thickness is more rapid for the screw component of the density that is in agreement with previous experimental observations of [11] but in contrast to theoretical results of [17], which predicted the limitative role of screw dislocations in the decrease due to their low mobility.

Thanks to the strict conformity to the selective etching, the described above XRD procedure has been applied to examine macrodistribution of the dislocations across the whole 2-inch diameter GaN-on-sapphire substrate. The variation of the total dislocation density with the distance from primary flat is shown in Figure 6. The W-shape distribution with the peaks at the center, in the middle, and at the periphery of the substrate along its radius is clearly seen. Resembling W-shape dislocation distributions have been routinely observed during the growth of bulk crystals, for example, GaAs, but along the crystal diameter with density peaking at the periphery and at the center of the boule [18]. Confirmed by simulations the $\mathrm{W}$-shape of the distribution in the bulk material is attributed to the plastic deformations caused by thermal stresses during after-growth cooling [19]. It is reasonable to suggest that the final dislocation pattern in $\mathrm{GaN}$ epilayers is defined by thermal stresses during growth and after-growth cooling. The thermal stresses can result in generation and multiplication of the dislocations when they exceed critical resolved shear stresses in $\mathrm{GaN}$ at elevated temperatures. They influence movement and interaction of the growth and threading dislocations and therefore are timedependent. Resulting from the steep temperature variations during epitaxial process the thermal stresses are the ones to be accounted for in the formation of the dislocation pattern in $\mathrm{GaN}$ epilayers.

\section{Summary}

It has been shown during the present study that the E-etching at elevated temperatures $\left(500-600^{\circ} \mathrm{C}\right)$ can be adopted for the dislocation etching in HVPE GaN epilayers. It has been suggested that the temperature increase (compared to Eetching of bulk GaN crystals) is caused by the compressive state of the epilayer grown on the thermally mismatched sapphire substrate.

It has been found that the XRD evaluation of the dislocation density in HVPE GaN epilayer based on the conventional Williamson-Hall plots and Dunn-Koch equation is in an excellent agreement with the results of the elevatedtemperature E-etching.

Application of the XRD procedure to the measurements of the dislocation distribution in 2-inch GaN-on-sapphire substrate suggests strongly the influence of the inelastic thermal stresses on the formation of the final dislocation pattern in the epilayer.

\section{Acknowledgment}

The authors would like to thank their present and former colleagues from the GaN Lab, Ostendo Technologies, Inc., for their continued support and ongoing interest in their research.

\section{References}

[1] R. Kuzel, "Kinematical diffraction by distorted crystalsdislocation X-ray line broadening," Zeitschrift für Kristallographie, vol. 222, no. 3-4, pp. 136-149, 2007.

[2] J. L. Weyher, M. Albrecht, T. Wosinski, G. Nowak, H. P. Strunk, and S. Porowski, "Study of individual grown-in and indentation-induced dislocations in GaN by defect-selective etching and transmission electron microscopy," Materials Science and Engineering B, vol. 80, pp. 318-321, 2001.

[3] J. L. Weyher, P. D. Brown, J. L. Rouvière, T. Wosinski, A. R. A. Zauner, and I. Grzegory, "Recent advances in defect-selective etching of GaN," Journal of Crystal Growth, vol. 210, no. 1, pp. 151-156, 2000.

[4] J. L. Weyher, L. Macht, G. Kamler, J. Borysiuk, and I. Grzegory, "Characterization of GaN single crystals by defect-selective etching," Physica Status Solidi C, vol. 0, no. 3, pp. 821-826, 2003.

[5] D. Zhuang and J. H. Edgar, "Wet etching of GaN, AlN, and SiC: a review," Materials Science and Engineering R, vol. 48, no. 1, pp. 1-46, 2005.

[6] M. Namerikawa, T. Sato, O. Takahashi, T. Suemasu, and F. Hasegawa, "Growth parameter dependence of HVPE GaN and 
polarity and crystal quality of the grown layers," Journal of Crystal Growth, vol. 237-239, no. 1-4, pp. 1089-1093, 2002.

[7] A. Koukitu, M. Mayumi, and Y. Kumagai, "Surface polarity dependence of decomposition and growth of GaN studied using in situ gravimetric monitoring," Journal of Crystal Growth, vol. 246, no. 3-4, pp. 230-236, 2002.

[8] K. Yamamoto, H. Ishikawa, T. Egawa, T. Jimbo, and M. Umeno, "EBIC observation of $\mathrm{n}-\mathrm{GaN}$ grown on sapphire substrates by MOCVD," Journal of Crystal Growth, vol. 189190, pp. 575-579, 1998.

[9] S. K. Mathisa, A. E. Romanov, L. F. Chena, G. E. Beltz, W. Pompe, and J. S. Speck, "Modeling of threading dislocation reduction in growing GaN layers," Journal of Crystal Growth, vol. 231, pp. 371-390, 2001.

[10] M. A. Moram and M. E. Vickers, "X-ray diffraction of IIInitrides," Reports on Progress in Physics, vol. 72, no. 3, Article ID 036502, 2009.

[11] J. Q. Liu, J. F. Wang, Y. F. Liu et al., "High-resolution X-ray diffraction analysis on HVPE-grown thick GaN layers," Journal of Crystal Growth, vol. 311, no. 10, pp. 3080-3084, 2009.

[12] S. Danis, V. Holy, Z. Zhong, G. Bauer, and O. Ambacher, "High-resolution diffuse x-ray scattering from threading dislocations in heteroepitaxial layers," Applied Physics Letters, vol. 85, no. 7, pp. 3065-3067, 2004.

[13] S. R. Lee, A. M. West, A. A. Allerman et al., "Effect of threading dislocations on the Bragg peakwidths of GaN, AlGaN, and AlN heterolayers," Applied Physics Letters, vol. 86, no. 24, Article ID 241904, 3 pages, 2005.

[14] G. K. Williamson and W. H. Hall, "X-ray line broadening from filed aluminium and wolfram," Acta Metallurgica, vol. 1, no. 1, pp. 22-31, 1953.

[15] M. B. Kerber, E. Schafler, and M. J. Zehetbauer, "Processing and evaluation of X-ray line profiles measured from nanostructured materials produced by severe plastic deformation," Reviews on Advanced Materials Science, vol. 10, pp. 427-433, 2005.

[16] C. G. Dunn and E. F. Koch, "Comparison of dislocation densities of primary and secondary recrystallization grains of Si-Fe," Acta Metallurgica, vol. 5, pp. 548-554, 1957.

[17] S. K. Mathis, A. E. Romanov, L. F. Chen, G. E. Beltz, W. Pompe, and J. S. Speck, "Modeling of threading dislocation reduction in growing GaN layers," Physica Status Solidi A, vol. 179, no. 1, pp. 125-145, 2000.

[18] D. L. Barrett, S. McGuigan, H. M. Hobgood, G. W. Eldridge, and R. N. Thomas, "Low dislocation, semi-insulating Indoped GaAs crystals," Journal of Crystal Growth, vol. 70, no. 1-2, pp. 179-184, 1984.

[19] N. Miyazaki, Y. Kuroda, and M. Sakaguchi, "Dislocation density analyses of GaAs bulk single crystal during growth process (Effects of crystal anisotropy)," Journal of Crystal Growth, vol. 218, no. 2, pp. 221-231, 2000. 

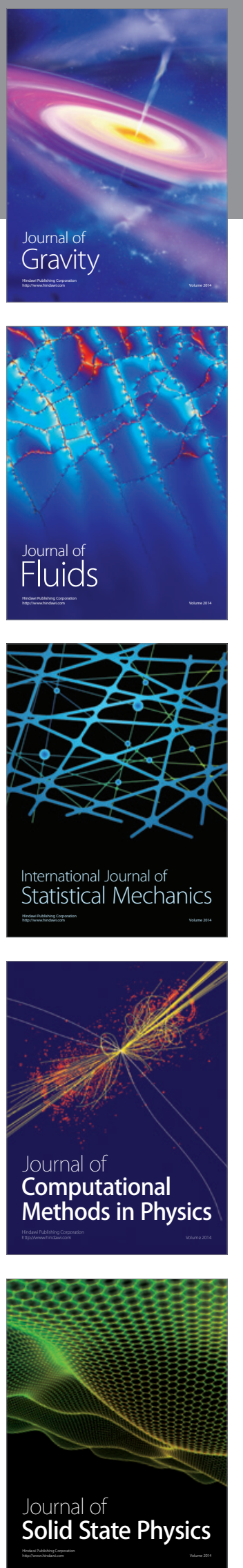
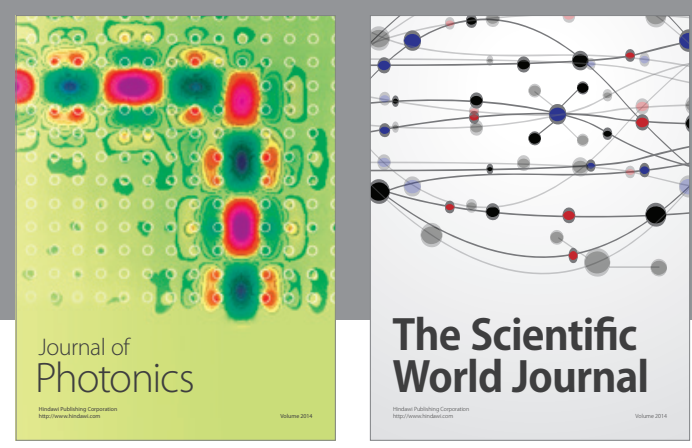

The Scientific World Journal

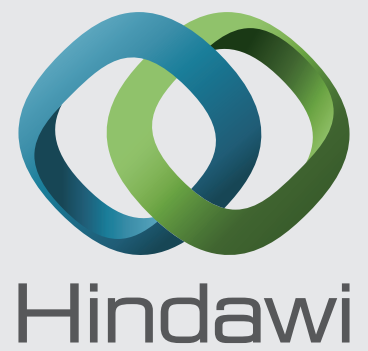

Submit your manuscripts at http://www.hindawi.com
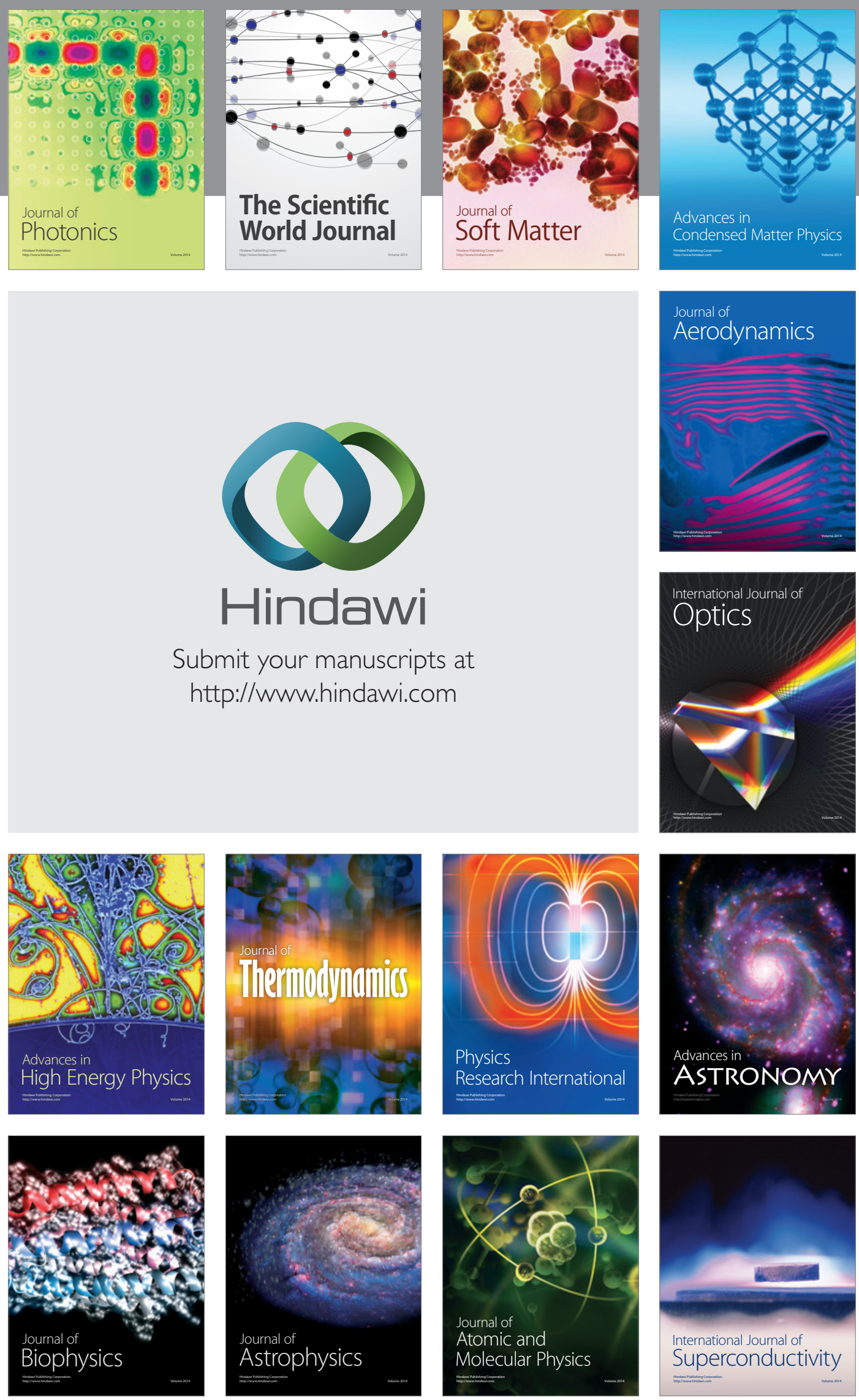
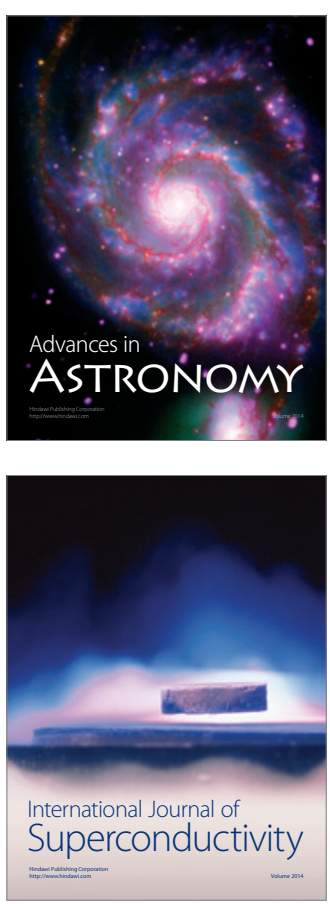